University of Nebraska - Lincoln

DigitalCommons@University of Nebraska - Lincoln

US Department of Energy Publications

U.S. Department of Energy

2005

\title{
Photoionization of Multiply Charged lons at the Advanced Light Source
}

A. S. Schlachter

Lawrence Berkeley National Laboratory, fsschlachter@lbl.gov

A. L. D. Kilcoyne

Lawrence Berkeley National Laboratory, ALKilcoyne@lbl.gov

A. Aguilar

Lawrence Berkeley National Laboratory, AAguilar@lbl.gov

M. F. Gharaibeh

University of Nevada - Reno

E. D. Emmons

University of Nevada - Reno

See next page for additional authors

Follow this and additional works at: https://digitalcommons.unl.edu/usdoepub

Part of the Bioresource and Agricultural Engineering Commons

Schlachter, A. S.; Kilcoyne, A. L. D.; Aguilar, A.; Gharaibeh, M. F.; Emmons, E. D.; Scully, S. W. J.; Phaneuf, R. A.; Müller, A. M.; Schippers, S.; Alvarez, I.; Cisneros, C.; Hinojosa, G.; and McLaughlin, B. M., "Photoionization of Multiply Charged lons at the Advanced Light Source" (2005). US Department of Energy Publications. 348.

https://digitalcommons.unl.edu/usdoepub/348

This Article is brought to you for free and open access by the U.S. Department of Energy at DigitalCommons@University of Nebraska - Lincoln. It has been accepted for inclusion in US Department of Energy Publications by an authorized administrator of DigitalCommons@University of Nebraska - Lincoln. 


\section{Authors}

A. S. Schlachter, A. L. D. Kilcoyne, A. Aguilar, M. F. Gharaibeh, E. D. Emmons, S. W. J. Scully, R. A. Phaneuf, A. M. Müller, S. Schippers, I. Alvarez, C. Cisneros, G. Hinojosa, and B. M. McLaughlin 


\title{
Photoionization of Multiply Charged Ions at the Advanced Light Source
}

\author{
A. S. Schlachter ${ }^{1}$, A. L. D. Kilcoyne ${ }^{1}$, A. Aguilar $^{2}$, M. F. Gharaibeh ${ }^{2}$, E. D. \\ Emmons $^{2}$, S. W. J. Scully ${ }^{2}$, R. A. Phaneuf ${ }^{2}$, A. Müller ${ }^{3}$, S. Schippers ${ }^{3}$, I. Alvarez ${ }^{4}$, \\ C. Cisneros ${ }^{4}$, G. Hinojosa ${ }^{4}$, and B. M. McLaughlin ${ }^{5}$ \\ ${ }^{I}$ Advanced Light Source, Lawrence Berkeley National Laboratory, Berkeley CA 94720, USA \\ ${ }^{2}$ Department of Physics, MS 220, University of Nevada, Reno NV 89557, USA \\ ${ }^{3}$ Institut für Atom- und Molekülphysik, Justus-Liebig-Universität, 35392 Giessen, Germany \\ ${ }^{4}$ Universidad Nacional Autonoma de Mexico, Cuernavaca, Mexico \\ ${ }^{5}$ Queen's University Belfast, BT71NN, UK
}

\begin{abstract}
Photoionization of multiply charged ions is studied using the merged-beams technique at the Advanced Light Source. An ion beam is created using a compact 10-GHz all-permanent-magnet ECR ion source and is accelerated with a small accelerator. The ion beam is merged with a photon beam from an undulator to allow interaction over an extended path. Absolute photoionization cross sections have been measured for a variety of ions along both isoelectronic and isonuclear sequences.
\end{abstract}

\section{INTRODUCTION}

Photoionization of ions is a fundamental process of importance in many high-temperature environments, such as in stars and nebulae, and in interstellar space. It is also an important process in hot dense laboratory plasmas, and of potential importance for modeling a plasma source for future lithography applications. There have been extensive theoretical calculations of cross sections for photoionization of ions, but few experiments to serve as benchmarks.

Absolute cross sections for photoionization of ions have been measured for many species of ions. Experiments are performed using a merged-beams end station at the Advanced Light Source (ALS). An ion beam from an electron-cyclotron-resonance (ECR) ion source is merged with an ultrabright beam of $\mathrm{x}$ rays from an undulator. Results are reported for several of the ion species studied to date, including measurement of lifetimes of K-shell vacancies in atomic carbon, observation of a truncated giant resonance in photoionization of $\mathrm{Ti}^{3+}$, and absolute photoionization cross-section measurements in $\mathrm{Sc}^{2+}$, metastable $\mathrm{O}^{+}$, and other ions.

\section{EXPERIMENTS}

Experiments have been conducted at undulator beamline 10.0 at the ALS, using the ion-photon merged-beams end station $[1,2]$ shown schematically in Fig 1. The technique was developed by Lyon and coworkers [3], and has been recently employed by Kjeldsen et al [4] and others [5]. An energy selected photon beam is merged over a path length of approximately one meter with an ion beam produced by an ECR ion source and a small accelerator.

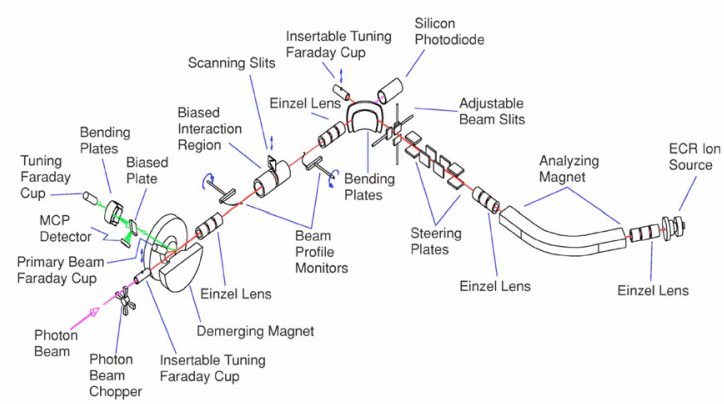

FIGURE 1. Schematic diagram of ion-photon mergedbeams end station. 
Ion beams are produced with a 10-GHz allpermanent-magnet ECR ion source based on a design by Trassl et al [6] shown schematically in Fig. 2. The ion source produces a high-intensity ion beam from either a gas or a metal evaporated in an oven inserted into the ion source. The ion beam in the interaction region is nearly parallel, has a typical diameter of 1-2 $\mathrm{mm}$, and has a current in the 1-100 nA range.

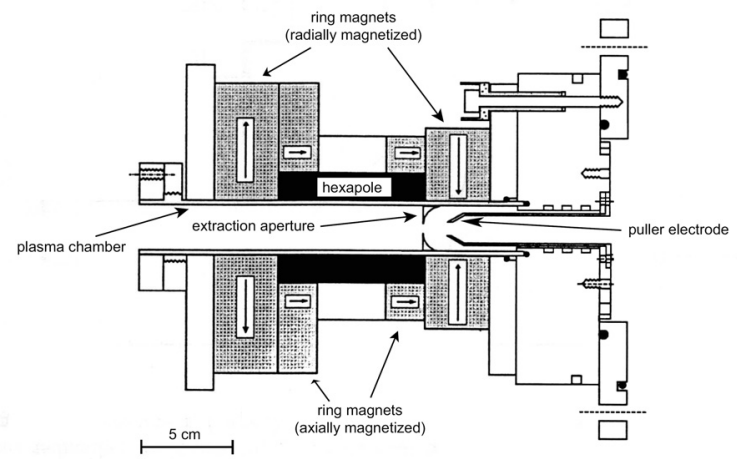

FIGURE 2. Schematic diagram of all-permanent-magnet ECR ion source [6].

The photon beam from an undulator is energy selected by a spherical-grating monochromator, and has a spectral resolving power in the range 1000 to 34,000 determined by the width of the entrance and exit slits of the monochromator. Typical photon flux is of the order of $10^{13}$ photons per second (an order of magnitude less at energies above $250 \mathrm{eV}$ ) for photons in the range 17 to $340 \mathrm{eV}$ at modest resolving power, and correspondingly less with increasing spectral resolution.

The ion beam and the counter-propagating photon beam are merged, and a potential is applied to a central $29.4-\mathrm{cm}$-long interaction region. This potential energylabels ions produced in the interaction region, thus providing a known interaction length. Downstream is an analyzing magnet which demerges the beams, separating ions which have changed charge from the primary ion beam. The current in the primary ion beam is measured in a Faraday cup, while the ions which have changed charge are counted using a singleparticle detector. The photon beam is mechanically chopped to allow separation of ions produced by collisions in background gas from photoions.

Two-dimensional intensity distributions of both beams are measured with rotating-wire beam-profile monitors and with a translating-slit scanner. Photon intensity is measured with a calibrated photodiode. The efficiency of the single-particle detector is measured in a separate experiment. All quantities required for measurement of an absolute cross section are thus measured.

\section{PHOTOIONIZATION OF METASTABLE ${ }^{+}$IONS}

An ECR ion source can produce a mixture of ground-state and metastable ions, making the analysis of photoionization spectra challenging. Absolute experimental measurements and theoretical calculations have been published [2] for cross sections for photoionization of $\mathrm{O}^{+}$ions from the ${ }^{2} \mathrm{P}^{\mathrm{o}}$ and ${ }^{2} \mathrm{D}^{\circ}$ metastable levels and from the ${ }^{4} \mathrm{~S}^{0}$ ground state in the photon energy range $30-35.5 \mathrm{eV}$. The measurements, reported by Covington et al [2] and by Aguilar et al [7] reveal a rich spectrum of resonances (Fig. 3) arising from $2 p-n s$ and $2 p$-nd transitions originating from ions in metastable states.

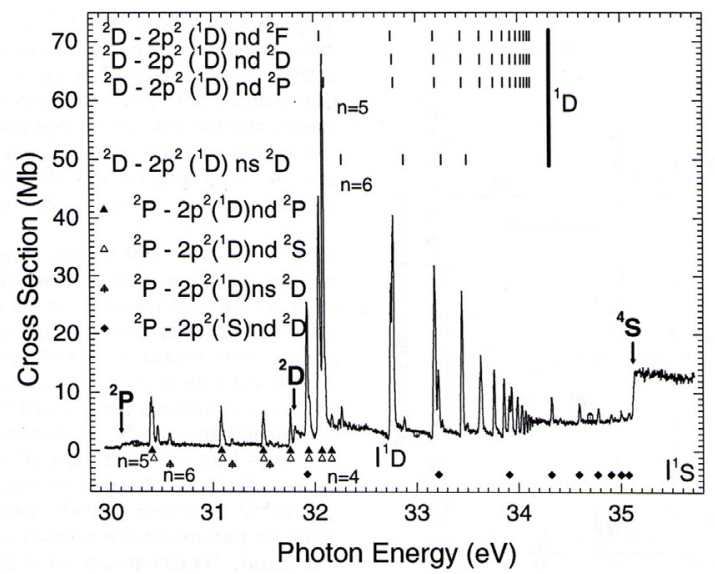

FIGURE 3. Experimental cross section for photoionization as a function of photon energy for an admixture of $57 \%$ metastable and $43 \%$ ground-state $\mathrm{O}^{+}$ions [2]. The energy resolution is $17 \mathrm{meV}$.

Comparison with theory shows that predicted resonance structure associated with the photoexcitation of autoionizing states is sensitive to the choice of basis functions.

\section{PHOTOIONIZATION OF $\mathrm{Sc}^{2+}$ IONS}

Cross sections for the photoionization of $\mathrm{Sc}^{2+}$ ions have been measured using the merged-beams apparatus at ALS as reported by Schippers et al [8]. The photon energy range 23-68 eV encompasses the direct $3 \mathrm{~d}$ and $3 \mathrm{p}$ photoionization thresholds. The experimental cross section is dominated by autoionizing resonances due to $3 p$ excitations 
predominantly decaying by Coster-Kronig and superCoster-Kronig transitions.

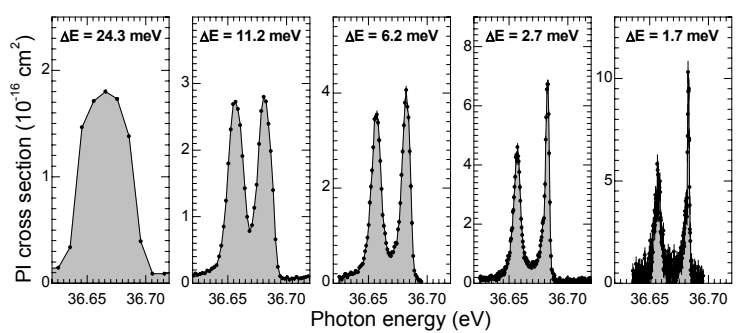

FIGURE 4. Influence of the experimental photon energy resolution on the measured photoionization cross section for a group of three resonances in photoionization of $\mathrm{Sc}^{2+}$ ions [8].

This system was studied with varying spectral resolution, shown in Fig 4: individual resonances were measured with a spectral resolution as high as 1.2 $\mathrm{meV}$, corresponding to a resolving power of nearly 34,000 . Although the effective ion target density is many orders of magnitude smaller, this resolution is comparable to that normally obtained in experiments with gas targets at ALS, illustrating the sensitivity of the merged-beams technique.

\section{PHOTOIONIZATION OF $\mathrm{C}^{2+}$ IONS}

Photoionization of an admixture of ${ }^{1} \mathrm{~S}$ ground-state and ${ }^{3} \mathrm{P}^{0}$ metastable-state $\mathrm{C}^{2+}$ ions has been studied [9].

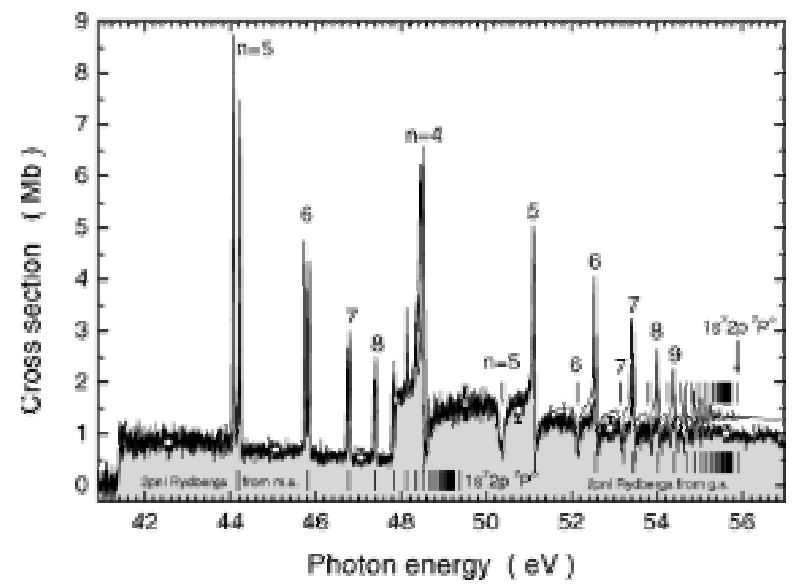

FIGURE 5. Measured and calculated cross sections for photoionization of $\mathrm{C}^{2+}$ ions [9]. Theory is shown by the curve; experimental results are shown as individual data points.

Theoretical cross sections were used to estimate that the primary ion beam consisted of $40 \%$ metastable ions. Figure 5 compares the experimental results with the results of an R-matrix theoretical calculation. The results are almost indistinguishable except at higher energies near the Rydberg-series limits.

\section{LIFETIME OF A K-SHELL VACANCY IN ATOMIC CARBON}

Lifetimes for K-shell vacancy states produced by removing a K-shell electron from atomic carbon have been determined by measurement of the natural linewidth of the $1 \mathrm{~s}-2 \mathrm{p}$ photoexcited states of $\mathrm{C}^{+}$ions [10]. The $\mathrm{K}$-shell vacancy states produced by direct $\mathrm{K}$ shell ionization of atomic carbon are identical to those produced by $1 \mathrm{~s}-2 \mathrm{p}$ photoexcitation of a $\mathrm{C}^{+}$ion. The vacancy states stabilize by emission of an electron to produce $\mathrm{C}^{2+}$.

Results are shown in Fig. 6 for photoexcitation of an admixture of ground-state and metastable $\mathrm{C}^{+}$ions. The metastable fraction was determined by use of theoretical cross sections calculated employing the Rmatrix method. Lifetime linewidths were determined by fitting a Voigt profile (a convolution of a Gaussian and a Lorentzian profile) to experimental results.

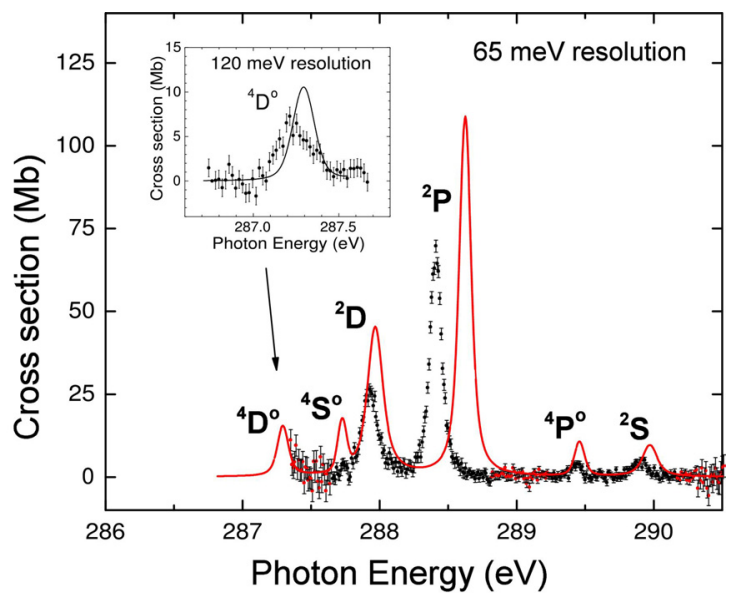

FIGURE 6. Experimental and theoretical cross sections for $1 \mathrm{~s}-2 \mathrm{p}$ photoexcitation of $\mathrm{C}^{+}$ions for an admixture of $80 \%$ ground-state and 20\% metastable-state ions [10].

Lifetimes are obtained from measured linewidths by use of the uncertainty principle. Lifetimes of K-shell vacancy states are found to be of the order of $10 \mathrm{fs}$, considerably longer than lifetimes observed for Kvacancy states in carbon-containing molecules, as 
there are additional L-shell electrons in molecules which are available to fill the K-shell vacancy.

\section{TRUNCATION OF A 'GIANT' DIPOLE RESONANCE IN PHOTOIONIZATION OF $\mathrm{Ti}^{3+}$ IONS}

Photoionization of triply charged titanium ions was studied using the merged-beams technique [11]. Cross sections are shown in Fig. 7. The energy range 42.6 to $49.4 \mathrm{eV}$ encompasses the threshold for photoionization of the $\mathrm{Ti}^{3+}$ ground state. A giant dipole resonance associated with $3 \mathrm{p}-3 \mathrm{~d}$ excitation in potassium-like ions decays by an extremely fast super-Coster-Cronig transition, and has a corresponding width of $1.5 \mathrm{eV}$. Since the central energy of this resonance in $\mathrm{Ti}^{3+}$ is located only $0.2 \mathrm{eV}$ above the ionization threshold, the low-energy part of the resonance is missing in the photoion yield spectrum, producing an unusual truncation of its lineshape.

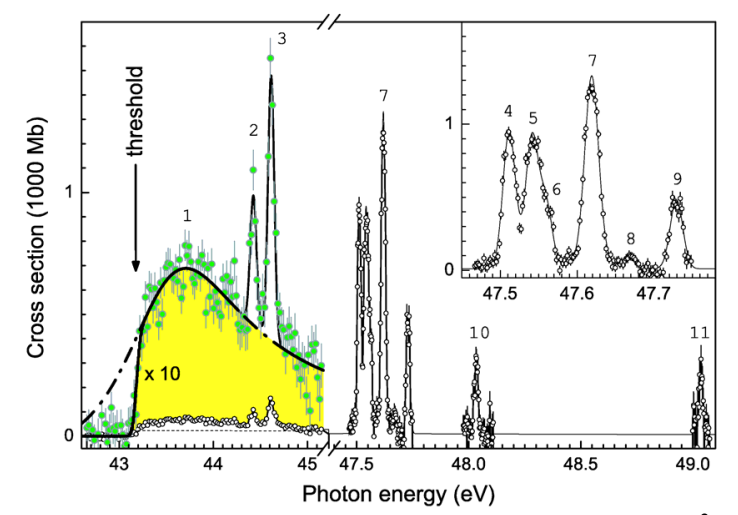

FIGURE 7. Cross section for photoionization of $\mathrm{Ti}^{3+}$ ions [11].

\section{THEORETICAL APPROACH}

R-matrix calculations have served a valuable role in guiding experiments at ALS, especially in locating resonances and regions of interest for experimental study. It is generally not possible to perform measurements over a large energy range with high spectral resolution to locate narrow resonances. Theoretical calculations were an invaluable guide in locating energy regions for measurement in the cases of K-shell photoionization of $\mathrm{C}^{+}$[10] and $\mathrm{C}^{2+}$ ions [12]. Theory has also been valuable in determining the metastable content of an ion beam from the ECR ion source, and in determining which resonances are attributable to ground-state ions and which to metastable ions.

\section{CONCLUSION}

The mating of an ECR ion source to the photon-ion end station at ALS makes possible systematic studies of photionization of ions along isoelectronic and isonuclear sequences. Examples of recent investigations at ALS are the potassium and nitrogen isoelectronic sequences, and the iron, xenon, and krypton isonuclear sequences. Photoionization measurements have also been initiated with singly and multiply charged ion beams of $\mathrm{C}_{60}$ and $\mathrm{C}_{70}$ produced in the ECR ion source [13].

\section{REFERENCES}

1. Covington, A. M et al, Phys. Rev. A 66, 062710 (2002) and references therein. Ph.D. Thesis, Alejandro AguilarMendoza, University of Nevada Reno (2003).

2. Covington, A. M. et al, Phys. Rev. Lett. 87, 243002 (2001).

3. Lyon I. C. et al, J. Phys. B 19, 4137 (1986).

4. Kjeldsen et al, J. Phys B 32, 4457 (1999).

5. Bizau, J.-M et al, Phys. Rev. Lett. 87, 273002 (2001); Itoh, Y. et al, J. Phys. B 28, 4733 (1995); Oura, M. et al, Nucl. Instrum. Meth. B 86, 190 (1994).

6. Trassl et al, Phys. Scr. T80, 504 (1999). Schlapp et al, Nucl. Instrum. Meth. B 98, 525 (1995), Trassl et al, Physica Scripta T73, 380 (1997).

7. Aguilar, A. et al, Astrophysical Journal Supplement Series 146, 129 (2003).

8. Schippers, S. et al, Phys. Rev A 67, 032702 (2003); Schippers, S. et al, Phys. Rev. Lett. 89, 193002 (2002).

9. Müller, A. et al, J. Phys. B 35, L137 (2002).

10. Schlachter, A. S. et al, J. Phys. B 37, L103 (2004).

11. Schippers, S.. et al, J.Phys. B 37, L209 (2004).

12. Scully, S. et al, article in preparation.

13. Scully, S. et al, article submitted for publication. 